\title{
AngiotensinII Preconditioning Promotes Angiogenesis In Vitro via ERKs Phosphorylation
}

\author{
Aili Guan, ${ }^{1}$ Yunzeng Zou, ${ }^{1,2}$ Hui Gong, ${ }^{2}$ Yuhong Niu, ${ }^{3}$ Yong Ye, ${ }^{2}$ Jianguo Jia, ${ }^{1}$ \\ Bingyu Li, ${ }^{1}$ Guoping Zhang, ${ }^{2}$ Sanli Qian, ${ }^{1}$ and Junbo Ge ${ }^{1,2}$ \\ ${ }^{1}$ Shanghai Institute of Cardiovascular Diseases, Zhongshan Hospital, Fudan University, 180 Feng Lin Road, Shanghai 200032, China
${ }^{2}$ Institutes of Biological Science, Fudan University, 138 Yixueyuan Road, Shanghai 200032, China
${ }^{3}$ Institute of Shanghai Medical Science and Technology Information, 602 West Jianguo Road, Shanghai 200032, China
}

Correspondence should be addressed to Yunzeng Zou, zou.yunzeng@zs-hospital.sh.cn

Received 26 September 2011; Revised 3 November 2011; Accepted 21 November 2011

Academic Editor: Yujian James Kang

Copyright (c) 2012 Aili Guan et al. This is an open access article distributed under the Creative Commons Attribution License, which permits unrestricted use, distribution, and reproduction in any medium, provided the original work is properly cited.

\begin{abstract}
AngiotensinII (AngII) is involved in not only the formation of cardiac hypertrophy but also the development of cardiac remodeling both of which are associated with myocardial angiogenesis. This study was therefore performed to clarify the effects of AngII on the formation of vasculatures by cultured cardiac microvascular endothelial cells (CMVECs) after a long-period stimulation with or without the AngII preconditioning. Incubation with AngII for $18 \mathrm{hrs}$ significantly impaired the formation of capillary-like tubes comparing to that without AngII. CMVECs with AngII pretreatment for 5 and 10 min formed more capillary-like tubes than those without AngII pretreatment, suggesting that preconditioning with AngII at a lower dose for a short period could prevent the further damage of CMVECs by a higher concentration of AngII. Moreover, AngII $\left(10^{-7} \mathrm{M}\right)$ stimulation for 5 and 10 min significantly induced the increase in extracellular signal-regulated protein kinases (ERKs) phosphorylation, and an ERKs inhibitor, PD98059, abrogated the increase in the formation of capillary-like tubes induced by the AngII-pretreatment. In conclusion, preconditioning with a lower concentration of AngII for a short period prevents the subsequent impairment of CMVECs by a higher dose of AngII, at least in part, through the increase in ERKs phosphorylation.
\end{abstract}

\section{Introduction}

Cardiac hypertrophy usually occurs as an adaptive response to increased workload to maintain cardiac function in early stages [1]. However, prolonged cardiac hypertrophy can lead to heart failure lately [2]. It has been postulated that a mismatch between the number of capillaries and the size of cardiomyocytes occurs during the development of cardiac hypertrophy and causes myocardial hypoxia [3, 4]. Various studies indicate that a potential relationship exists among cardiac angiogenesis, cardiac hypertrophy, and cardiac function [5-7]. Furthermore, rennin-angiotensin systems (RASs), especially angiotensinII(AngII), play a crucial role in the development of cardiac hypertrophy $[8,9]$. AngII is proved to be related with myocardial cell apoptosis and remodeling [10-12]. Though both dysfunctions of angiogenesis in myocardium and AngII are critical to the progress of cardiac hypertrophy and heart failure [13], roles of AngII in myocardial angiogenesis at the different stages during the pathogenic process are unclear. The aim of this study was to identify effects of AngII on the formation of vasculatures by cultured cardiac microvascular endothelial cells (CMVECs) at an early or later time point after stimulation.

\section{Materials and Methods}

2.1. Cell Culture and AngiotensinII Treatment. Primary rat cardiac microvascular endothelial cells (CMVECs) were obtained from male Wistar rats (8-9 weeks old) by the method of planting myocardial tissue $[14,15]$ and were cultured in high-glucose Dulbecco's modified Eagle's medium (DMEM) (Gibco, USA) containing 20\% fetal bovine serum (FBS, Hyclone), $100 \mathrm{u} / \mathrm{mL}$ penicillin, and $100 \mathrm{u} / \mathrm{mL}$ streptomycin (Sigma, USA). The 2nd and 3rd rat CMVECs were seeded on a 6 -well plate at $5 \times 10^{5} /$ well and cultured for 


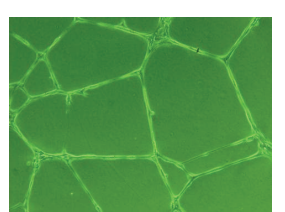

Control

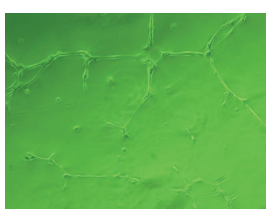

$0 \mathrm{~min}$

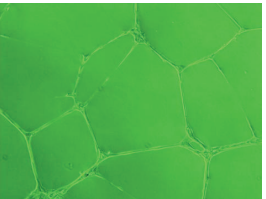

$5 \mathrm{~min}$

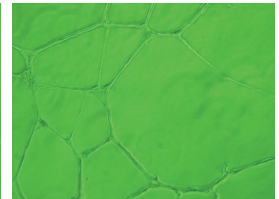

$10 \mathrm{~min}$ AngiotensinII $10^{-7} \mathrm{M}$
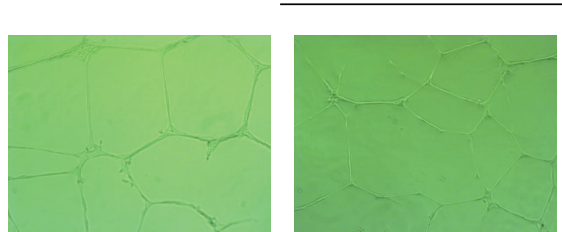

30 min

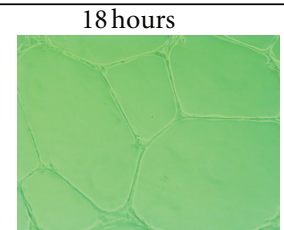

AngiotensinII $10^{-6} \mathrm{M}$

$$
15 \mathrm{~min}
$$

$60 \mathrm{~min}$

AngiotensinII $10^{-7} \mathrm{M}$

(a)

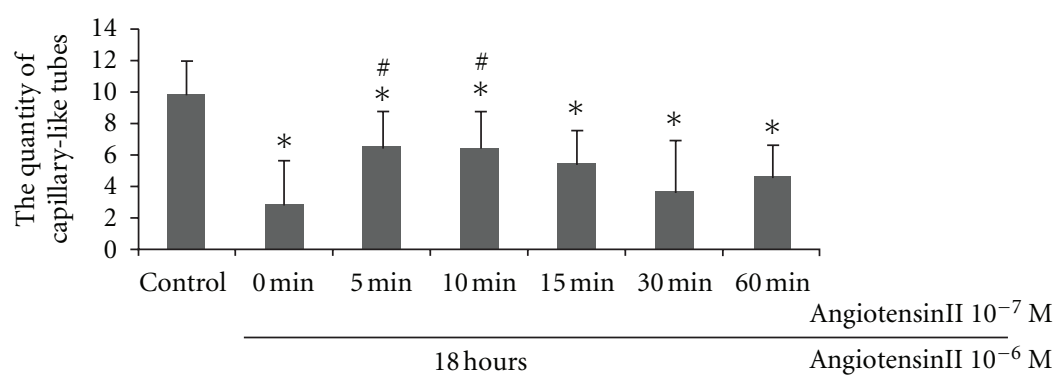

(b)

Figure 1: Time-dependent effects of AngII preconditioning on AngII-induced impairment of capillary-like tube formation in cultured CMVECs. CMVECs were pretreated with AngII $\left(10^{-7} \mathrm{M}\right)$ for the indicated times and then seeded onto the matrigel in plates containing AngII $\left(10^{-6} \mathrm{M}\right)$ or not (control). Eighteen hrs later, the formation of capillary-like tubes was observed under an optical microscope. (a) Representative photomicrographs of capillary-like tubes (100xmagnification). (b) Quantitative analysis for capillary-like tube formation. Capillary-like tubes were counted in randomly selected 5 fields for each plate. Data represent mean \pm SD obtained from 15 independent experiments $(n=15) ;{ }^{*} P<0.05$ versus control group; ${ }^{\#} P<0.05$ versus the group without AngII pretreatment $(0 \mathrm{~min})$.

24 hours. And 24 hours later, cells were cultured in serumfree DMEM for another 24 hours and then were incubated by AngII $\left(10^{-7} \mathrm{M}\right)$ for $5,10,15,30$, and $60 \mathrm{~min}$, respectively. Finally, CMVECs were collected and lysed for the further western blotting analysis and capillary-like tube formation analysis.

2.2. PD98059 Treatment. The 2nd and 3rd CMVECs were seeded on a 6 -well plate at $5 \times 10^{5} /$ well and cultured for 24 hours. Then, they were cultured in serum-free DMEM for another 24 hours, incubated by PD $98059(50 \mu \mathrm{M})$ for $30 \mathrm{~min}$ (DMSO as controls) and AngII $\left(10^{-7} \mathrm{M}\right)$ for a further $10 \mathrm{~min}$. Finally, cells were collected and lysed for the further western blotting analysis and capillary-like tube formation analysis

2.3. Western Blotting Analysis. Total protein was extracted from the cultured cells and then electrophoresed in $12 \%$ polyacrylamide gel and transferred to PVDF membrane. The expression of extracellular signal-regulated protein kinases (ERKs) phosphorylation was detected using a Phosphop44/42 MAPK Rabbit mAb (1:1,000; Cell Signaling). The membrane was then incubated with peroxidase-conjugated rabbit anti-goat $\operatorname{IgG}(1: 5,000$; Jackson) and HRP-conjugated monoclonal mouse antiglyceraldehyde-3-phosphate dehydrogenase (GAPDH, KangChen, China). Immunoreactive proteins were subjected to an ECL detection system (GE healthcare, RPN2106).

2.4. Capillary-Like Tube Formation. $200 \mu \mathrm{L}$ growth-factorreduced matrigel matrix (BD company) was homogenized and layered into a 24-well plate on a cooled planar surface and allowed to solidify at $37^{\circ} \mathrm{C}$. CMVECs that AngII $\left(10^{-7} \mathrm{M}\right)$ pretreated or PD98059 pretreated were detached with trypsin EDTA, resuspended in high-glucose DMEM, and supplemented with $1 \%$ of FBS and AngII $\left(10^{-6} \mathrm{M}\right)$. And suspension was seeded into plates at $5 \times 10^{4}$ cells/well and cultivated at $37^{\circ} \mathrm{C}$ atmosphere with $5 \% \mathrm{CO}_{2} / 95 \%$ air for 18 hours. Images of tubes were taken at 100xmagnification with a digital output camera attached to an inverted phasecontrast microscope (Leica. Germany), and five random fields of view were counted to analyze the relative number of capillary-like tubes.

2.5. Statistical Analysis. All the data were presented as mean \pm SD and were analyzed using ANOVA tests (least significant 


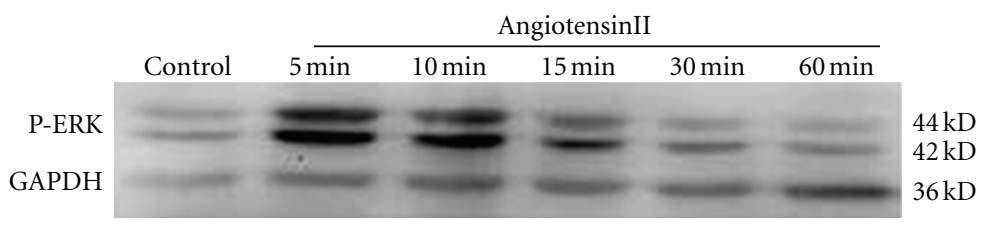

(a)

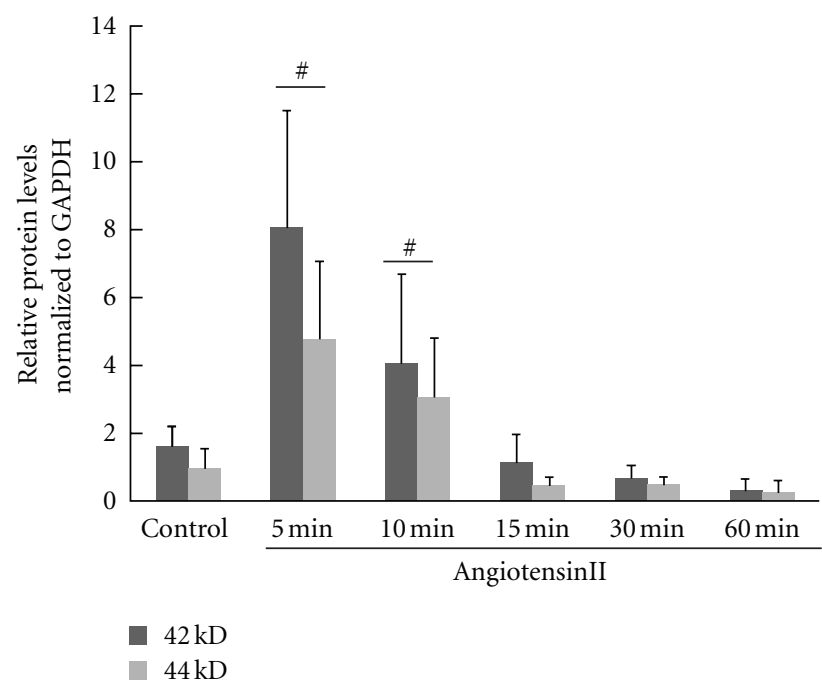

(b)

Figure 2: Time-dependent effects of AngII on ERKs phosphorylation in cultured CMVECs. CMVECs were treated with AngII (10 ${ }^{-7}$ M) for the indicated times and then collected and lysed for western blot analysis. (a) Representative immunoblots showing the expression of phosphor ERKs. GAPDH was used as a loading control. (b) Quantitative analysis for the expression of phosphor ERKs. Data represent mean \pm SD obtained from 6 independent experiments $(n=6) ;{ }^{*} P<0.05$ versus control group $(0 \mathrm{~min})$.

difference $t$-tests) for multiple-means comparison. A twotailed $P$ value of $<0.05$ was considered statistically significant. All statistical analysis was performed by SPSS 16.0 for Windows (SPSS Inc., Chicago, IL).

\section{Results}

3.1. Impairment of CMVECs to Form Capillary-Like Tubes and the Protective Effect of AngII Pretreatment. In order to investigate the effect of AngII on CMVECs capillary-like tube formation, we select the function of its capillary-like tube formation to observe. The results showed that stimulation of cultured CMVECs with AngII $\left(10^{-6} \mathrm{M}\right)$ for 18 hours impaired its capillary-like tube formation, and cells that either AngII $\left(10^{-7} \mathrm{M}\right)$ pretreated for 5 or $10 \mathrm{~min}$ formed more capillary-like tubes than those without AngII pretreatment (Figures 1(a) and 1(b)), suggesting that precondition with AngII at a lower dose for a short period could prevent the subsequent impairment of CMVECs by a higher dose of AngII.

3.2. Phosphorylation of ERKs in Cultured CMVECs following AngII Stimulation. In order to identify the effect of AngII on ERKs phosphorylation in CMVECs, we observed levels of ERKs phosphorylation using western blotting analysis. The figures showed that ERKs phosphorylation in CMVECs under AngII $\left(10^{-7} \mathrm{M}\right)$ stimulation for 5 and $10 \mathrm{~min}$ was significantly increased more than those without AngII addition (Figures 2(a) and 2(b)).

3.3. ERKs Phosphorylation Participated in the Protective Effect by Preconditioning. The above results pointed out that pretreated CMVECs with AngII for 5 or 10 min could protect the subsequent impairment induced by a higher dose of AngII (Figure 1), and interestingly, levels of ERKs phosphorylation raised at that stimulation point as Figure 2 reveals. Therefore, we hypothesized that ERKs phosphorylation might take part in the protective effect by preconditioning. PD98059, an ERKs inhibitor, was selected to inhibit ERKs phosphorylation, and function of capillary-like tube formation was observed in CMVECs. Results indicated that $50 \mu \mathrm{M}$ PD98059 could inhibit ERKs phosphorylation to the last penny (Figures 3(a) and 3(b)), and the number of capillarylike tubes of CMVECs significantly decreased when inhibited ERKs phosphorylation (Figures 4(a) and 4(b)). The figures suggested that preconditioning with a lower concentration of AngII for a short period prevents the subsequent impairment of CMVECs by a higher dose of AngII through the increase of ERKs phosphorylation. 


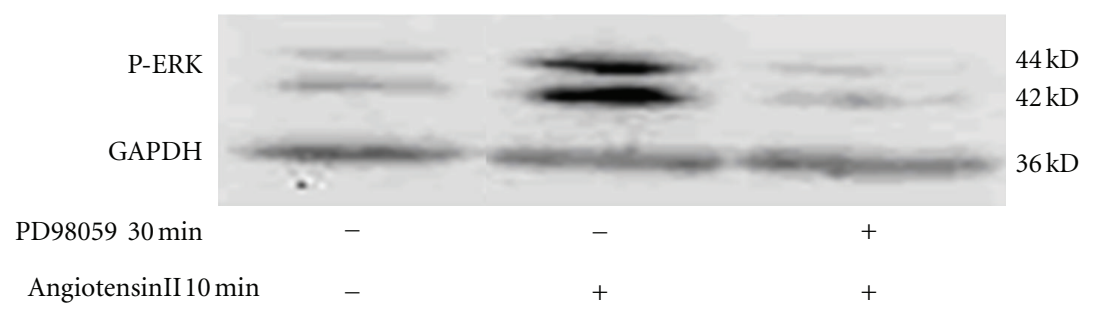

(a)

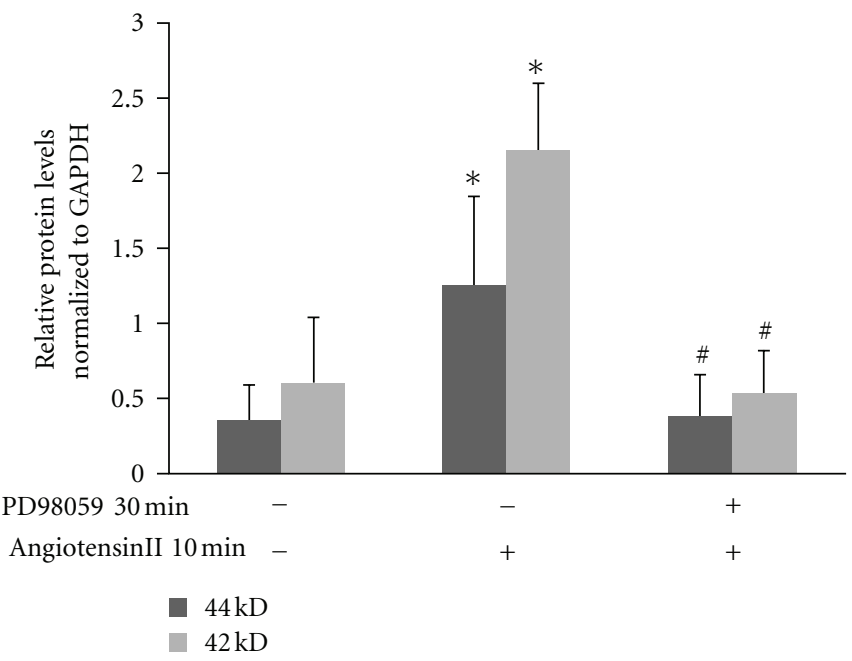

(b)

FIGURE 3: The effects of PD98059 on ERKs phosphorylation in cultured CMVECs. CMVECs were incubated with (+) or without $(-)$ PD98059 $(50 \mu \mathrm{M})$, an ERKs inhibitor, for $30 \mathrm{~min}$ and then treated with $(+)$ or without $(-)$ AngII $\left(10^{-7} \mathrm{M}\right)$ for $10 \mathrm{~min}$. Thereafter, the cells were collected and lysed for western blotting. (a) Representative immunoblots showing the expression of phosphor ERKs. GAPDH was used as a loading control. (b) Quantitative analysis for the expression of phosphor ERKs. Data represent mean \pm SD obtained from 4 independent experiments $(n=4) .{ }^{*} P<0.05$ versus CMVECs with neither PD98059 nor AngII treatment; ${ }^{\#} P<0.05$ versus CMVECs without PD98059 but with AngII treatment.

\section{Discussion}

In normal mammalian myocardium, each cardiomyocyte is surrounded by an intricate network of capillaries and is next to cardiac microvascular endothelial cells (CMVECs) [16]. The unique structure between CMVECs and cardiomyocyte determines that CMVECs may participate in regulating cardiomyocyte function. In mature myocardium, CMVECs not only supply cardiomyocytes with oxygen and nutrient but also promote cardiomyocytes organization and survival with local protective signals. Cardiomyocytes cocultured with CMVECs may promote marked cardiomyocyte reorganization along the EC structures and inhibit cardiomyocytes apoptosis and necrosis [17, 18]. So, CMVECs are very important in the heart. A reduction of capillary length density has been found in several experimental models of cardiac hypertrophy [19]. In particular, major roles were shown for VEGF, angiopoietin, and Tie2 in cardiac vasculogenesis and angiogenesis after AngII infusion [20]. In the present study, our data provides that AngII incubated for 18 hours could damage functions of CMVECs capillary-like tube formation, AngII $\left(10^{-7} \mathrm{M}\right)$ pretreatment for $5 \sim 10 \mathrm{~min}$ could diminish the subsequent impairment induced by a higher dose of AngII incubation. And the effect was related to ERKs phosphorylation which was considered to be one signal related to cell proliferation. Since the similar protective effect of AngII by preconditioning in ischemia-reperfusion models had been approved [21-29], we did not observe the preconditioning effect in perfusion heart here. Though the detailed mechanisms underlying ERKs phosphorylation in function of capillary-tube formations and other various pathological angiogenesis remain to be fully investigated, and the active preconditioning effect might be difficult to be realized in organism, our data had unraveled that this important molecular ERKs phosphorylation maintaining proliferation participated in the process of protection for CMVECs to form capillary-like tubes. Since AngII -induced angiogenesis is a critical physiological or pathological event in diverse cardiac diseases, these findings might be broadly applicable and significant in the therapeutics of cardiac diseases such as hypertension, acute myocardial infarction, and chronic systolic heart failure, particularly as how to explain that ACEI/ARB combination therapy was associated with more adverse effects without any increase in benefit. In 


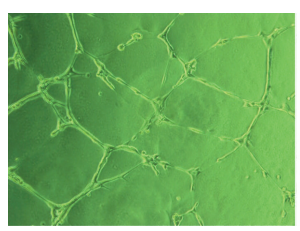

Control
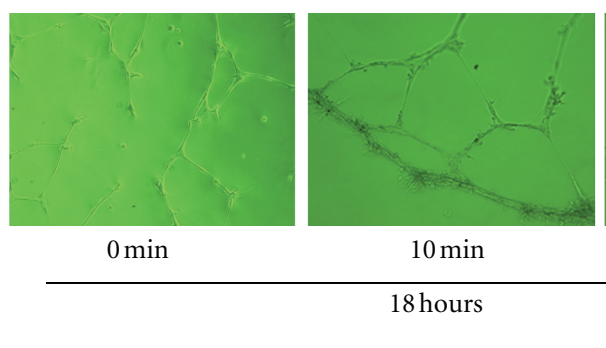

(a)

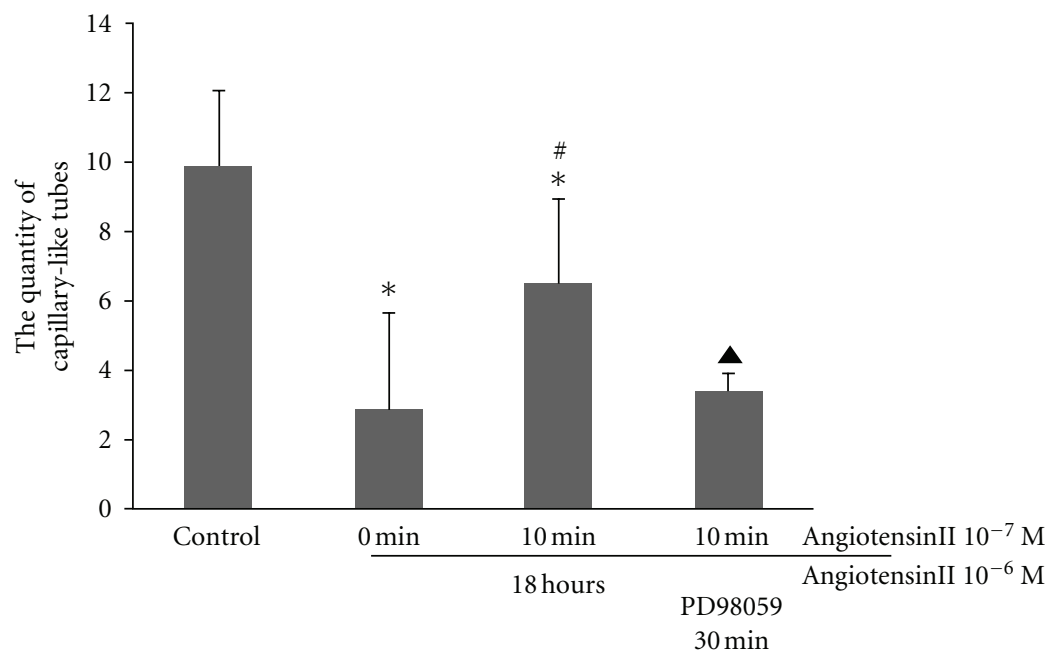

(b)

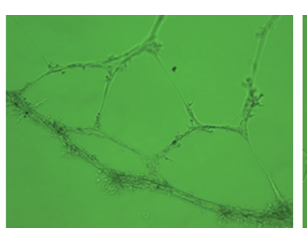

$10 \mathrm{~min}$

PD9805
$30 \mathrm{~min}$

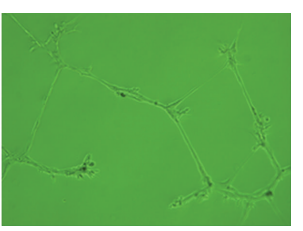

AngiotensinII $10^{-7} \mathrm{M}$ AngiotensinII $10^{-6} \mathrm{M}$

FIgURE 4: Effects of ERKs inhibition on AngII preconditioning-induced improvement of vasculature formation under the condition of AngII stimulation. CMVECs preincubated with or without PD98059 $(50 \mu \mathrm{M})$ for 30 min were treated with $10^{-7} \mathrm{M}$ AngII for the indicated times, and then the cells were seeded onto the matrigel in plates containing AngII $\left(10^{-6} \mathrm{M}\right)$ or not (control). Eighteen hrs later, the formation of capillary-like tubes was observed under an optical microscope. (a) Representative photomicrographs of capillary-like tubes (100xmagnification). (b) Quantitative analysis for capillary-like tube formation. Capillary-like tubes were counted in randomly selected 5 fields for each plate. Data represent mean \pm SD obtained from 12 independent experiments $(n=12)$. ${ }^{*} P<0.05$ versus control group; ${ }^{\#} P<0.05$ versus the group without AngII pretreatment $(0 \mathrm{~min}) ; \boldsymbol{\Delta} P<0.05$ versus the group pretreated with AngII (10 min) but without PD98059.

other words, that appropriate inhibition of AngII rather than complete inhibition of AngII is benefit of cardiac diseases.

\section{Acknowledgments}

The authors would like to thank Drs. Zhen Ma and Yuemei Chen for their technical support. This study was undertaken in Shanghai Institute of Cardiovascular Diseases. The study work was supported by the National Basic Research Program of China (2007CB512003).

\section{References}

[1] N. Frey and E. N. Olson, "Cardiac hypertrophy: the good, the bad, and the ugly," Annual Review of Physiology, vol. 65, pp. 45-79, 2003.

[2] D. Levy, R. J. Garrison, D. D. Savage, W. B. Kannel, and W. P. Castelli, "Prognostic implications of echocardiographically determined left ventricular mass in the framingham heart study," The New England Journal of Medicine, vol. 322, no. 22, pp. 1561-1566, 1990.
[3] M. L. Marcus, S. Koyanagi, and D. G. Harrison, "Abnormalities in the coronary circulation that occur as a consequence of cardiac hypertrophy," American Journal of Medicine A, vol. 75, no. 3, pp. 62-66, 1983.

[4] R. J. Tomanek, "Response of the coronary vasculature to myocardial hypertrophy," Journal of the American College of Cardiology, vol. 15, no. 3, pp. 528-533, 1990.

[5] F. J. Giordano, H. P. Gerber, S. P. Williams et al., "A cardiac myocyte vascular endothelial growth factor paracrine pathway is required to maintain cardiac function," Proceedings of the National Academy of Sciences of the United States of America, vol. 98, no. 10, pp. 5780-5785, 2001.

[6] K. G. Shyu, J. Y. Liou, B. W. Wang, W. J. Fang, and H. Chang, "Carvedilol prevents cardiac hypertrophy and overexpression of hypoxia-inducible factor- $1 \alpha$ and vascular endothelial growth factor in pressure-overloaded rat heart," Journal of Biomedical Science, vol. 12, no. 2, pp. 409-420, 2005.

[7] Y. S. Yoon, S. Uchida, O. Masuo et al., "Progressive attenuation of myocardial vascular endothelial growth factor expression is a seminal event in diabetic cardiomyopathy: restoration of microvascular homeostasis and recovery of cardiac function in diabetic cardiomyopathy after replenishment of local vascular 
endothelial growth factor," Circulation, vol. 111, no. 16, pp. 2073-2085, 2005.

[8] H. Yue, W. Li, R. Desnoyer, and S. S. Karnik, "Role of nuclear unphosphorylated STAT3 in angiotensin II type 1 receptorinduced cardiac hypertrophy," Cardiovascular Research, vol. 85, no. 1, pp. 90-99, 2010.

[9] J. F. Ainscough, M. J. Drinkhill, A. Sedo et al., "Angiotensin II type-1 receptor activation in the adult heart causes blood pressure-independent hypertrophy and cardiac dysfunction," Cardiovascular Research, vol. 81, no. 3, pp. 592-600, 2009.

[10] A. Leri, P. P. Claudio, Q. Li et al., "Stretch-mediated release of angiotensin II induces myocyte apoptosis by activating p53 that enhances the local renin-angiotensin system and decreases the Bcl-2-to-Bax protein ratio in the cell," The Journal of Clinical Investigation, vol. 101, no. 7, pp. 1326-1342, 1998.

[11] A. Leri, Y. Liu, P. P. Claudio et al., "Insulin-like growth factor- 1 induces $\mathrm{Mdm} 2$ and down-regulates p53, attenuating the myocyte renin-angiotensin system and stretch-mediated apoptosis," American Journal of Pathology, vol. 154, no. 2, pp. 567-580, 1999.

[12] Y. Liu, A. Leri, B. Li et al., "Angiotensin II stimulation in vitro induces hypertrophy of normal and postinfarcted ventricular myocytes," Circulation Research, vol. 82, no. 11, pp. 11451159, 1998.

[13] M. Sano, T. Minamino, H. Toko et al., "p53-induced inhibition of Hif- 1 causes cardiac dysfunction during pressure overload," Nature, vol. 446, no. 7134, pp. 444-448, 2007.

[14] X. H. Wang, S. F. Chen, H. M. Jin, and R. M. Hu, "Differential analyses of angiogenesis and expression of growth factors in micro- and macrovascular endothelial cells of type 2 diabetic rats," Life Sciences, vol. 84, no. 7-8, pp. 240-249, 2009.

[15] F. M. Marelli-Berg, E. Peek, E. A. Lidington, H. J. Stauss, and R. I. Lechler, "Isolation of endothelial cells from murine tissue," Journal of Immunological Methods, vol. 244, no. 1-2, pp. 205215, 2000.

[16] D. L. Brutsaert, "Cardiac endothelial-myocardial signaling: its role in cardiac growth, contractile performance, and rhythmicity," Physiological Reviews, vol. 83, no. 1, pp. 59-115, 2003.

[17] D. A. Narmoneva, R. Vukmirovic, M. E. Davis, R. D. Kamm, and R. T. Lee, "Endothelial cells promote cardiac myocyte survival and spatial reorganization: implications for cardiac regeneration," Circulation, vol. 110, no. 8, pp. 962-968, 2004.

[18] S. Lecht, C. Foerster, H. Arien-Zakay, C. Marcinkiewicz, P. Lazarovici, and P. I. Lelkes, "Cardiac microvascular endothelial cells express and release nerve growth factor but not fibroblast growth factor-2," In Vitro Cellular and Developmental Biology, vol. 46, no. 5, pp. 469-476, 2010.

[19] G. Mall, G. Zimmer, S. Baden, and T. Mattfeldt, "Capillary neoformation in the rat heart-stereological studies on papillary muscles in hypertrophy and physiologic growth," Basic Research in Cardiology, vol. 85, no. 6, pp. 531-540, 1990.

[20] P. Carmeliet, "Mechanisms of angiogenesis and arteriogenesis," Nature Medicine, vol. 6, no. 4, pp. 389-395, 2000.

[21] G. W. Booz, J. N. Day, and K. M. Baker, "Interplay between the cardiac renin angiotensin system and JAK-STAT signaling: role in cardiac hypertrophy, ischemia/reperfusion dysfunction, and heart failure," Journal of Molecular and Cellular Cardiology, vol. 34, no. 11, pp. 1443-1453, 2002.

[22] S. Das, R. M. Engelman, N. Maulik, and D. K. Das, "Angiotensin preconditioning of the heart: evidence for redox signaling," Cell Biochemistry and Biophysics, vol. 44, no. 1, pp. 103-110, 2006.
[23] R. J. Diaz and G. J. Wilson, "Selective blockade of AT1 angiotensin II receptors abolishes ischemic preconditioning in isolated rabbit hearts," Journal of Molecular and Cellular Cardiology, vol. 29, no. 1, pp. 129-139, 1997.

[24] A. J. Ferreira, R. A. Santos, and A. P. Almeida, "Angiotensin(1-7): cardioprotective effect in myocardial ischemia/ reperfusion," Hypertension, vol. 38, no. 3, part 2, pp. 665-668, 2001.

[25] A. Nakano, T. Miura, N. Ura, K. Suzuki, and K. Shimamoto, "Role of the angiotensin II type 1 receptor in preconditioning against infarction," Coronary Artery Disease, vol. 8, no. 6, pp. 343-350, 1997.

[26] A. Sharma and M. Singh, "Effect of ethylisopropyl amiloride, a $\mathrm{Na}+\mathrm{H}+$ exchange inhibitor, on cardioprotective effect of ischaemic and angiotensin preconditioning," Molecular and Cellular Biochemistry, vol. 214, no. 1-2, pp. 31-38, 2000.

[27] A. Sharma and M. Singh, "Possible mechanism of cardioprotective effect of angiotensin preconditioning in isolated rat heart," European Journal of Pharmacology, vol. 406, no. 1, pp. 85-92, 2000.

[28] X. H. Xiao and D. G. Allen, "The role of endogenous angiotensin II in ischaemia, reperfusion and preconditioning of the isolated rat heart," Pflugers Archiv European Journal of Physiology, vol. 445, no. 6, pp. 643-650, 2003.

[29] Y. Liu, A. Tsuchida, M. V. Cohen, and J. M. Downey, "Pretreatment with angiotensin II activates protein kinase C and limits myocardial infarction in isolated rabbit hearts," Journal of Molecular and Cellular Cardiology, vol. 27, no. 3, pp. 883892, 1995. 


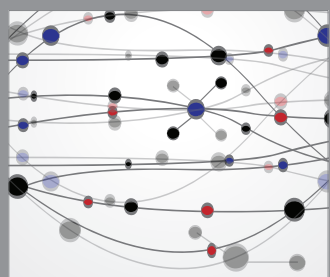

The Scientific World Journal
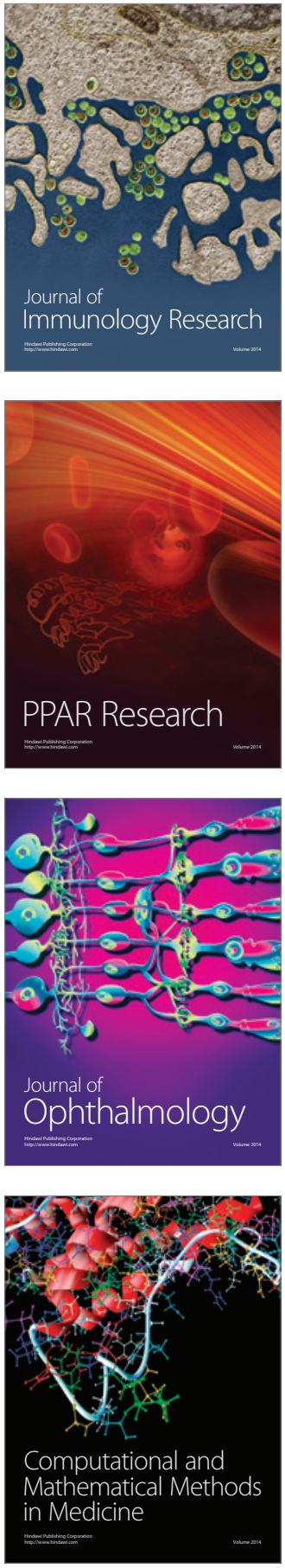

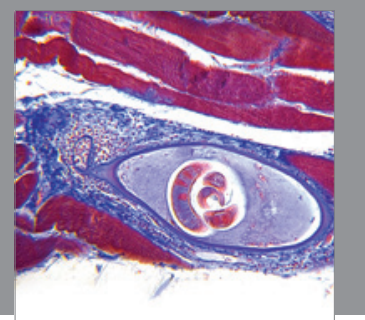

Gastroenterology

Research and Practice
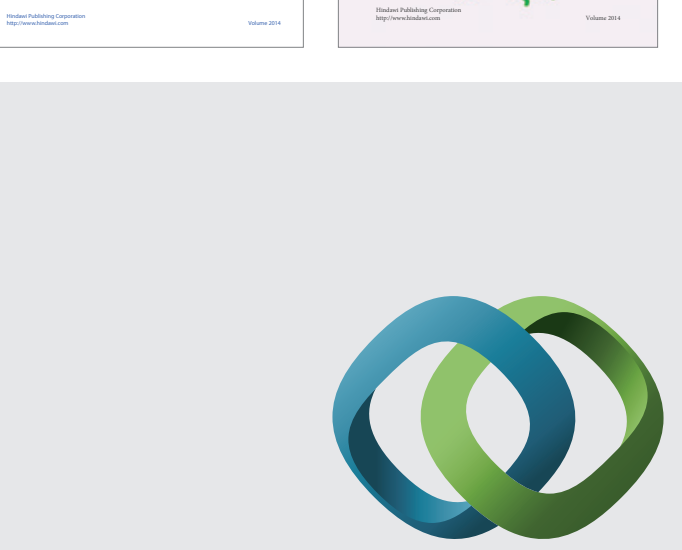

\section{Hindawi}

Submit your manuscripts at

http://www.hindawi.com
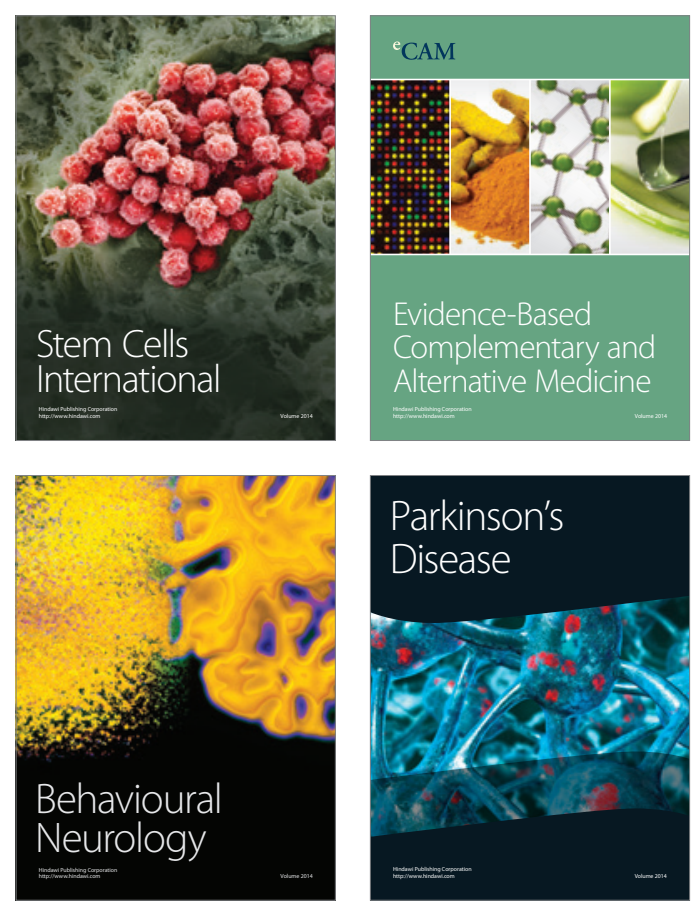

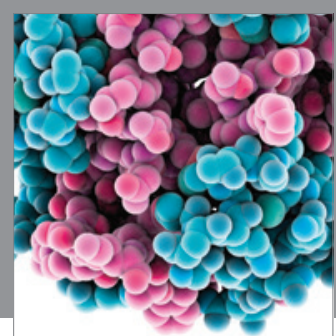

Journal of
Diabetes Research

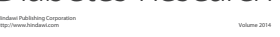

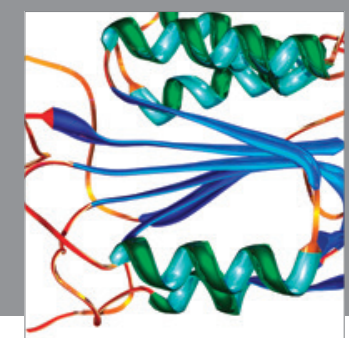

Disease Markers
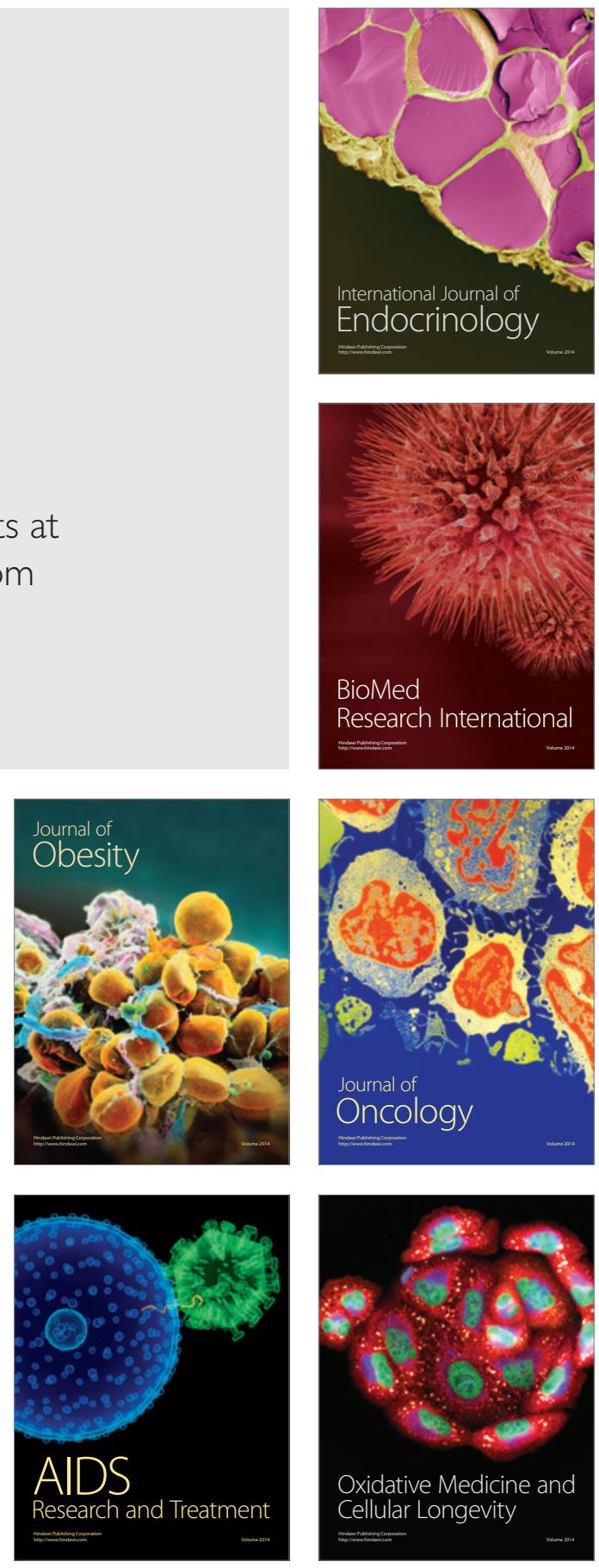